\title{
C-reactive protein as a screening test for gestational diabetes mellitus in first-trimester of pregnancy: a prospective cohort study
}

\author{
Tarek A. Farghaly ${ }^{1}$, Neveen A. Helmy², Ahmed M. Abbas ${ }^{1 *}$, Abdel Ghaffar M. Ahmed ${ }^{1}$
}

\begin{abstract}
${ }^{1}$ Department of Obstetrics and Gynecology, Faculty of Medicine, Assiut University, Assiut, Egypt
${ }^{2}$ Department of Obstetrics and Gynecology, Sahel Selim Hospital, Assiut, Egypt
\end{abstract}

Received: 11 October 2018

Accepted: 27 October 2018

*Correspondence:

Dr. Ahmed M. Abbas,

E-mail: bmr90@hotmail.com

Copyright: () the author(s), publisher and licensee Medip Academy. This is an open-access article distributed under the terms of the Creative Commons Attribution Non-Commercial License, which permits unrestricted non-commercial use, distribution, and reproduction in any medium, provided the original work is properly cited.

\section{ABSTRACT}

Background: The current study aims to determine if increased inflammation identified by increased C-reactive protein (CRP) level is associated with the subsequent development of gestational diabetes mellitus (GDM).

Methods: A prospective cohort study conducted at a tertiary University Hospital included 496 booking for their antenatal care visit between 10-12 weeks of gestation. They were tested for presence of CRP in blood. All women without a history of antenatal type 1 or type 2 diabetes undergo routine GDM screening with the 50-g oral glucoseloading test (GLT) between 24 and 28 weeks 'gestation. Women whose 1-h post-loading plasma glucose level $>7.8$ $\mathrm{mmol} / \mathrm{l}$ (>140 mg/dl) undergo a diagnostic, fasting, 100-g, 3-h oral glucose tolerance test (OGTT) within 1-2 weeks following the GLT.

Results: Among these 496 women, a total of 27 women (5.4\%) were diagnosed as GDM and 10 women $(2.0 \%)$ were diagnosed as IGT at week 24-28. The CRP was positive and higher in the GDM diagnosed group ( $\mathrm{p}=0.000)$ compared to those who did not develop GDM. CRP was positively correlated with diagnosis of GDM ( $\mathrm{r}=0.438, \mathrm{p}=0.012)$.

Conclusions: There is an association between first-trimester inflammation, marked by increased CRP levels and subsequent risk of GDM.

Keywords: C-reactive protein, Gestational diabetes mellitus, Glucose tolerance test

\section{INTRODUCTION}

Gestational diabetes mellitus (GDM) is defined as a condition of glucose intolerance that begins or first recognized during pregnancy. ${ }^{1,2}$ The incidence of GDM is rising, parallel to the increase in maternal age, type II diabetes and obesity prevalence. ${ }^{3}$ The Hyperglycemia and adverse pregnancy outcomes (HAPO) study confirmed that hyperglycemia in pregnancy increases the risk of adverse maternal, fetal and neonatal outcomes. ${ }^{4,5}$ Diagnosing women with GDM early provides the opportunity to reduce adverse event rates, as shown in two studies: the Australian carbohydrate intolerance study in pregnant women (ACHOIS) and the Maternal-
Fetal Medicine Units Network treatment of mild gestational diabetes (MFMUN-GDM) study. ${ }^{6,7}$

Prediction of GDM in early pregnancy has been the subject of many studies with the aim of initiating early treatment and lifestyle changes. ${ }^{8-10}$ An increased serum level of C-reactive protein (CRP), an inflammation marker, has been shown to be associated with insulin resistance. Furthermore, increased inflammation at baseline has been reported to be an independent risk factor for development of GDM. ${ }^{9}$ Although some investigators have developed good negative predictors for GDM, most of these factors had poor positive predictive value and limited efficacy. ${ }^{11}$ 
Currently, most guidelines recommend an oral glucose tolerance test (OGTT) between 24 and 28 gestational weeks as the method of diagnosis for GDM. ${ }^{12-16}$ According to the definition, GDM can develop at any time during pregnancy; however, most GDM cases are diagnosed after 24 weeks. Following diagnosis, proper management (glycemic monitoring, lifestyle changes, nutritional counseling, exercise and insulin use if appropriate, etc.) of GDM in pregnant women will be beneficial in controlling maternal and neonatal short-term complications.

However, offspring of mothers with GDM are still at a higher risk for developing diabetes, obesity and metabolic disorders in the long term. ${ }^{17-20}$ One potential reason is that these offspring were exposed to maternal hyperglycemia prior to diagnosis. Therefore, current guidelines have noticed that early diagnosis of GDM may improve maternal, fetal and neonatal outcomes. Although most of the guidelines recommend an OGTT at 24-28 gestational weeks, none provided robust evidence for the reason behind this time point.

The current study aims to determine the value of firsttrimester CRP measurement as a screening test for GDM.

\section{METHODS}

\section{Inclusion criteria}

- The study included all healthy pregnant women attending the Antenatal care clinic in Women Health Hospital, Assiut, Egypt over a period of 12 months from January to December 2017.

\section{Exclusion criteria}

- Patients were excluded if they had any of the following: preexisting chronic medical conditions that may affect acute phase markers, including DM, polycystic ovarian syndrome, collagen vascular diseases, inflammatory bowel disease, and preeclampsia.

- Moreover, those on current use of corticosteroids were also excluded.

The institutional ethical review board approved the study protocol and all women signed a written consent before participation in the study.

Study tools: Authors included 496 healthy pregnant women reporting for antenatal care in the first trimester during the first prenatal visit, (which was typically between 10- and 12-weeks' gestation) they were tested for presence of CRP in blood, in addition to the standard antenatal tests. Complete history and examination had been done to exclude any case having disease affecting CRP. Non-fasting serum samples collected at the conclusion of the first prenatal visit from women who provided informed consent to participate in the study.
Specific GDM risk factors were assessed including age, ethnicity, personal history of GDM, previous delivery of macrosomic infant and family history of GDM or macrosomic infant or type 2 DM. Baselines data collected include age, past medical and obstetric history, medications, smoking and blood pressure. Blood pressure, urine analysis and other laboratory tests results recorded at each subsequent prenatal visit.

CRP was tested by the latex agglutination semiquantitative test kit. This test kit is standardized to detect CRP concentrations of approximately $6 \mathrm{mg} / \mathrm{L}$ or higher in undiluted serum samples (positive test). All women without a history of antenatal type 1 or type 2 diabetes undergo routine GDM screening with the $50-\mathrm{g}$ oral glucose-loading test (GLT) between 24 and 28 weeks 'gestation, however any case discovered later on during routine antenatal care included in the study.

In the non-fasting state, subjects consume $50 \mathrm{~g}$ of oral glucose beverage and glucose levels will be assayed in 1$\mathrm{h}$ post-loading plasma samples. Women whose 1-h postloading plasma glucose level $>7.8 \mathrm{mmol} / \mathrm{l}$ (> $140 \mathrm{mg} / \mathrm{dl})$ had been considered to be at increased risk for GDM and undergo a diagnostic, fasting, 100-g, 3-h oral glucose tolerance test (OGTT) within 1-2 weeks following the GLT. Glucose levels assayed in baseline fasting plasma samples and in three post-challenge plasma, samples collected at 1-h intervals. The 100-g OGTT performed in the morning after overnight fast. Venous blood samples drawn at baseline and 60,120, and $180 \mathrm{~min}$. following ingestion of a standard 100-g glucose load.

\section{Glycemic status}

The OGTT stratifies participants into three glycemic tolerance groups:

1. GDM, as defined by the National Diabetes Data Group (NDDG) criteria (requires at least two of the following: fasting glucose, $>5.8 ; 1 \mathrm{~h}$ post-challenge (pc) glucose, $>10.6 ; 2 \mathrm{~h} \mathrm{pc},>9.2$; or $3 \mathrm{~h} \mathrm{pc}$, $>8.1 \mathrm{mmol}$ ).

2. Impaired glucose tolerance (IGT), as defined by NDDG criteria (requires one of above GDM criteria); and

3. Normal glucose tolerance (NGT), defined as subjects not meeting any of the GDM or IGT criteria.

\section{Statistical analysis}

Date entry and data analysis were done using SPSS version 22 (Statistical Package for Social Science). Data were presented as number, percentage, mean, standard deviation and median. Chi-square test and Fisher Exact test were used to compare between qualitative variables. Mann-Whitney test was used to compare quantitative variables between two groups. Spearman correlation was done to measure correlation between quantitative variables in case of non-parametric data. Med-Calc 
Program was used to calculate sensitivity, specificity, PPV, NPV, accuracy and AUC. P-value considered statistically significant when $\mathrm{p}<0.05$.

\section{RESULTS}

A total of 508 women were eligible for the study. Four women were excluded as they were lost to follow-up (due to choice of prenatal care in a different hospital). An additional eight women were withdrawn as they experienced pregnancy loss before reaching week 24 . Finally, 496 pregnant women were recruited for the statistical analysis. Among these 496 women, a total of 27 women $(5.4 \%)$ met the ACOG criteria and were diagnosed as GDM and 10 women (2.0\%) were diagnosed as IGT at week 24-28. Table 1 shows personal and obstetrical data of all cases. Out of 496 cases, 235 cases $(47.4 \%)$ were $>25$ years old, 131 cases $(26.4 \%)$ were $25->30$ years old and 130 cases $(26.2 \%)$ were $<30$ years old. There were 198 cases primigravida.
Table 1: Baseline characteristics of the study participants.

\begin{tabular}{|lll|}
\hline Characteristics & No. $(\mathbf{n = 4 9 6 )}$ & $\%$ \\
\hline Age (years) & & \\
\hline$<25$ & 235 & 47.4 \\
\hline $25-<30$ & 131 & 26.4 \\
\hline$>30$ & 130 & 26.2 \\
\hline Mean \pm SD & $25.99 \pm 5.66$ & \\
\hline Gravidity (mean \pm SD) & $2.49 \pm 1.67$ \\
\hline Parity (mean \pm SD) & $2.00 \pm 1.18$ & \\
\hline Nullipara & 198 & 39.9 \\
\hline 1 & 126 & 25.4 \\
\hline $2-3$ & 139 & 28.0 \\
\hline$>3$ & 33 & 6.7 \\
\hline Abortions (mean \pm SD) & $0.45 \pm 0.76$ & \\
\hline Previous CS (mean \pm SD) & $0.82 \pm 0.86$ & \\
\hline Consanguinity & & \\
\hline Yes & 69 & 13.9 \\
\hline No & 427 & 86.1 \\
\hline
\end{tabular}

Table 2: The difference between groups according to the baseline characteristics.

\begin{tabular}{|c|c|c|c|c|c|c|}
\hline & GDM (n=27) & IGT (n=10) & Normal $(n=459)$ & \multirow{2}{*}{ P-value $^{1}$} & \multirow{2}{*}{ P-value ${ }^{2}$} & \multirow{2}{*}{ P-value } \\
\hline & Mean \pm SD & Mean士SD & Mean \pm SD & & & \\
\hline Age (years) & $36.74 \pm 4.24$ & $33.00 \pm 4.50$ & $25.20 \pm 4.97$ & $0.043^{*}$ & $0.000^{*}$ & $0.000 *$ \\
\hline Gravidity & $5.30 \pm 2.46$ & $4.20 \pm 1.55$ & $2.29 \pm 1.43$ & 0.117 & $0.000 *$ & $0.000 *$ \\
\hline Parity & $3.48 \pm 1.38$ & $2.67 \pm 0.87$ & $1.85 \pm 1.08$ & 0.093 & $0.000^{*}$ & $0.007^{*}$ \\
\hline Abortions & $1.57 \pm 1.20$ & $0.89 \pm 1.05$ & $0.34 \pm 0.61$ & 0.134 & $0.000 *$ & $0.044^{*}$ \\
\hline Previous CS & $1.04 \pm 1.07$ & $1.11 \pm 0.78$ & $0.79 \pm 0.84$ & 0.610 & 0.305 & 0.188 \\
\hline \multicolumn{4}{|c|}{ Consanguinity } & \multirow{3}{*}{0.869} & \multirow{3}{*}{$0.001 *$} & \multirow{3}{*}{$0.027 *$} \\
\hline Yes & $10(37.0 \%)$ & $4(40.0 \%)$ & $55(12.0 \%)$ & & & \\
\hline No & $17(63.0 \%)$ & $6(60.0 \%)$ & $404(88.0 \%)$ & & & \\
\hline
\end{tabular}

GDM: gestational diabetes mellitus; IGT: impaired glucose tolerance; CS: cesarean section; SD: standard deviation;

*: statistical significant difference; ${ }^{1}$ : Comparison between GDM and IGT; ${ }^{2}$ : Comparison between GDM and normal; ${ }^{3}$ : Comparison between IGT and normal.

Table 3: The difference between groups according to the GDM risk factors.

\begin{tabular}{|c|c|c|c|c|c|c|c|c|c|}
\hline \multirow{2}{*}{ Risk factors } & \multicolumn{2}{|c|}{ GDM $(n=27)$} & \multicolumn{2}{|c|}{ IGT (n=10) } & \multicolumn{2}{|c|}{ Normal $(n=459)$} & \multirow{2}{*}{ P-value ${ }^{1}$} & \multirow{2}{*}{ P-value ${ }^{2}$} & \multirow{2}{*}{ P-value ${ }^{3}$} \\
\hline & No. & $\%$ & No. & $\%$ & No. & $\%$ & & & \\
\hline Family history of DM & 15 & 55.6 & 2 & 20.0 & 55 & 12.0 & 0.073 & $0.000 *$ & 0.348 \\
\hline Past history of macrosomia & 11 & 40.7 & 6 & 60.0 & 13 & 2.8 & 0.460 & $0.000 *$ & $0.000 *$ \\
\hline Past history of IUFD & 4 & 14.8 & 0 & 0.0 & 3 & 0.7 & 0.557 & $0.000 *$ & 0.798 \\
\hline Past history of neonatal death & 6 & 22.2 & 0 & 0.0 & 9 & 2.0 & 0.162 & $0.000 *$ & 0.655 \\
\hline Past history of gestational diabetes & 5 & 18.5 & 0 & 0.0 & 4 & 0.9 & 0.295 & $0.000 *$ & 0.767 \\
\hline
\end{tabular}

GDM: gestational diabetes mellitus; IGT: impaired glucose tolerance; IUFD: intrauterine fetal death; *: statistical significant difference;

${ }^{1}$ : Comparison between GDM and IGT; ${ }^{2}$ : Comparison between GDM and normal; ${ }^{3}$ : Comparison between IGT and normal.

There was a statistical significant difference at age, gravidity, parity, number of previous abortions and consanguinity between GDM cases and normal cases. GDM was more likely to develop in older age, higher gravidity, multiparity and those with recurrent abortions (Table 2). Table 3 shows a statistical significant difference between cases of GDM and normal cases at all risk factors including family history of DM, past history of macrosomic baby, past history of IUFD, past history of neonatal death and past history of GDM $(p=.000)$. On the other hand, there was no statistical significant difference between cases of GDM and cases of IGT at all risk factors. Table 4 shows a statistical significant difference between GDM cases and normal cases at the rate of 
glycosuria and positive CRP at the first antenatal visit $(\mathrm{p}=.000)$. The same observed with women with IGT cases versus normal cases (p.022 and .000, respectively). No statistical significant difference between GDM cases and IGT cases regarding both tests.

Table 4: Data collected at the first visit and their statistical significance.

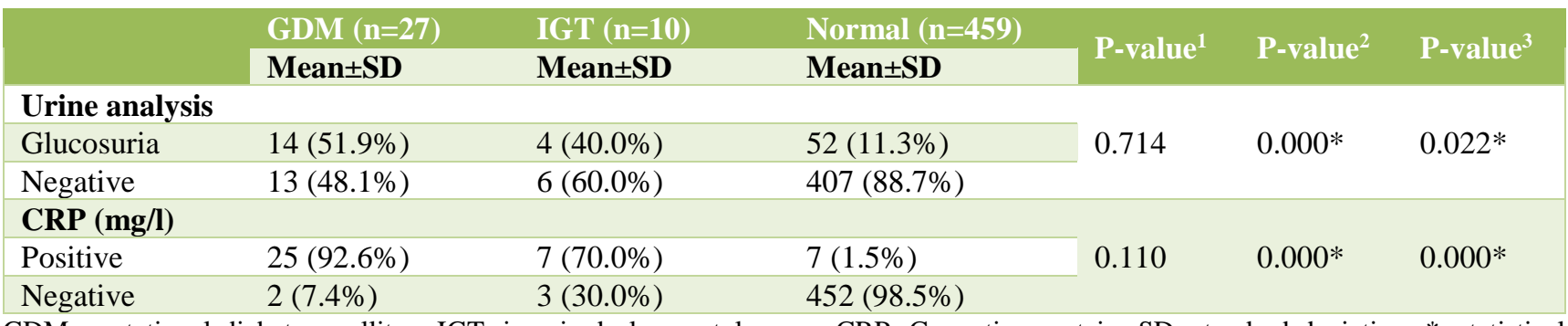

GDM: gestational diabetes mellitus; IGT: impaired glucose tolerance; CRP: C-reactive protein; SD: standard deviation; *: statistical significant difference; ${ }^{\text {: }}$ : Comparison between GDM and IGT; ${ }^{2}$ : Comparison between GDM and normal; ${ }^{3}$ : Comparison between IGT and normal.

Table 5: Data collected at the second visit and their statistical significance.

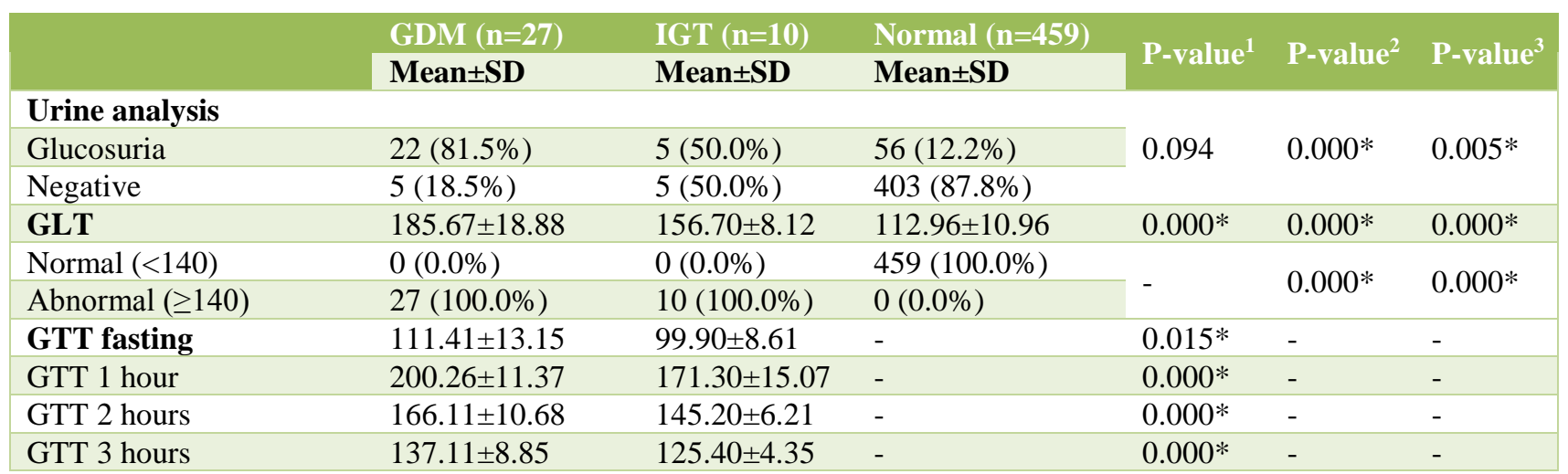

GDM: gestational diabetes mellitus; IGT: impaired glucose tolerance; SD: standard deviation; GLT: glucose loading test; GTT: glucose tolerance test; *: statistical significant difference; ${ }^{1}$ : Comparison between GDM and IGT; ${ }^{2}$ : Comparison between GDM and normal; ${ }^{3}$ : Comparison between IGT and normal.

Table 6: Correlation analysis between different variables and CRP levels.

\begin{tabular}{|lll|} 
& \multicolumn{2}{c|}{ CRP (mg/l) } \\
\hline Age (years) & 0.569 & P-value \\
\hline GLT & 0.767 & $0.000^{*}$ \\
\hline GTT fasting & 0.250 & $0.000^{*}$ \\
\hline GTT 1 hour & 0.438 & 0.167 \\
\hline GTT 2 hours & 0.578 & $0.012^{*}$ \\
\hline GTT 3 hours & 0.271 & $0.001^{*}$ \\
\hline
\end{tabular}

CRP: C-reactive protein; GLT: glucose loading test; GTT: glucose tolerance test; *: statistical significance.

The results of urine analysis, GLT and GTT at the second visit were presented in Table 5. There was a statistical significant higher rate of glucosuira in GDM and IGT cases versus normal cases $(\mathrm{p}=0.000$ and 0.005 respectively). The results of GLT and GTT were significantly higher in GDM cases than IGT cases.

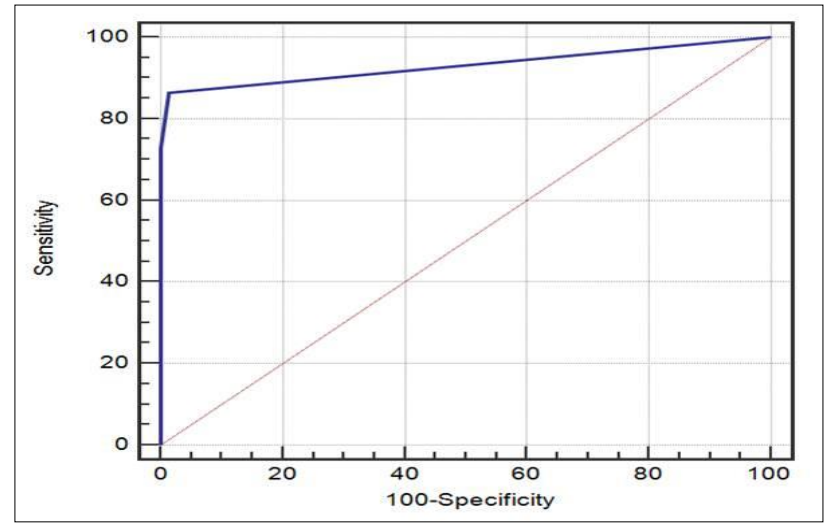

Figure 1: Receiver operating characteristic curve analysis of the predictive value of CRP for prediction of gestational diabetes mellitus.

CRP was positively strong correlated with GLT ( $\mathrm{r}=0.767$, $\mathrm{p}=0.000$ ) and moderately correlated with GTT 1 and 2 
hours. Moreover, the level of CRP with positively moderate correlated with participants' age $(r=0.569$, $\mathrm{p}=0.000)$ (Table 6).

According to ROC curve analysis, the sensitivity of CRP is $86.49 \%$, specificity $98.47 \%$, accuracy $97.58 \%$ and area under curve (AUC) is 0.930 at cut-off $>5$ for detection of GDM (Figure 1).

\section{DISCUSSION}

CRP in the first trimester is associated with subsequent development of GDM in pregnancy. Performing CRP in the first trimester is easy and can help identify patients who have a high risk of later developing GDM. Early intervention for these patients may lead to a good perinatal outcome. The present study revealed significant differences in qualitative and semi quantitative serum CRP levels between the cases of GDM as compared to normal cases $(p<0.0005)$, and that diagnostics strength of qualitative serum CRP was acceptable. Present study was in accordance with the findings of $\mathrm{Li}$ et al, Liu et al and Rota et al. ${ }^{21-23}$ In their study they found that serum CRP level was higher in the women with GDM as compared to normal pregnant women. Qiu et al and Myles Wolf et al also found that CRP was higher in patients of GDM compared to normal pregnant women. ${ }^{24,25}$ In the patients of GDM, there is increased expression of genes for chronic stress and inflammatory pathways. This leads to increase in production of CRP. ${ }^{26}$

A study by Ahi et al found also quantitative CRP test is acceptable in predicting gestational diabetes. ${ }^{27}$ On the contrary, a study by Mohan, 2006 found that CRP in early months as an inflammatory marker is not a dependable screening test for gestational diabetes developing in later months of pregnancy, however this study applied only on 91 women. ${ }^{28}$ In GDM, TNF- $\alpha$ is another inflammatory marker was found to be strongest predictor of insulin resistance in pregnancy. ${ }^{29}$ This TNF- $\alpha$ is responsible for production and release of CRP from the liver. ${ }^{30}$ GDM show features of insulin resistance and it has been proved by many studies that CRP level is increased in insulin resistance syndrome. ${ }^{31}$

Despite 4 decades of research, a general consensus has not been reached about optimal approach to screening for gestational diabetes. The important challenge is in the choice between general or elective screening, and also diagnostic threshold of GCT. ${ }^{32}$ Cunningham et al reported sensitivity and specificity of GCT test in screening for glucose with $140 \mathrm{mg} / \mathrm{dl}$ threshold were: $80 \%$ and $82-86 \%$ respectively, and with $130 \mathrm{mg} / \mathrm{dl}$ threshold, $90 \%$ and $75-80 \%$, respectively. ${ }^{32}$ In the present study, the sensitivity and specificity of CRP test were found to be $86.49 \%$ and $98.47 \%$, respectively. Positive and negative predicting values of qualitative CRP test in predicting gestational diabetes were estimated at $82.1 \%$ and $98.9 \%$, respectively, without considering any gestational diabetes risk factors, which is indicative of acceptable diagnostic strength. Area under the curve was found 0.930 . In a study by Syngelaki et al reported in pregnancies that develop GDM no evidence of an inflammatory response at 11-13 weeks' gestation and the levels of serum TNF-á and Hs-CRP are not useful in firsttrimester screening for GDM. ${ }^{33}$ In another study by Corcoran et al., 2016 reported that higher levels of CRP were significantly associated with macrosomia but hSCRP did not demonstrate additional predictive ability for GDM. ${ }^{34}$

Finally, following further and more comprehensive studies to assess diagnostic strength of serum CRP in pregnant women at risk of GDM, use of CRP test can be considered in pregnant women qualified for gestational diabetes risk factors, as a new, fast and reliable screening test.

\section{CONCLUSION}

In this prospective study authors identified an association between first-trimester inflammation, marked by increased CRP levels, and subsequent risk of GDM. CRP could be a useful screening test in the first-trimester for prediction of GDM.

\section{Funding: No funding sources}

Conflict of interest: None declared

Ethical approval: The study was approved by the Institutional Ethics Committee

\section{REFERENCES}

1. National Institutes of Health consensus development conference statement: diagnosing gestational diabetes mellitus, March 4-6. Obstet Gynecol. 2013;122(Pt 1):358-69.

2. Landon MB, Spong CY, Thom E, Carpenter MW, Ramin SM, Casey B, et al. A multicenter, randomized trial of treatment for mild gestational diabetes. $\mathrm{N}$ Engl J Med. 2009;361:1339-48.

3. Beischer NA, Wein P, Sheedy MT, Steffen B. Identification and treatment of women with hyperglycaemia diagnosed during pregnancy can significantly reduce perinatal mortality rates. Aust N Z J Obstet Gynaecol. 1996;36(3):239-47.

4. HAPO Study Cooperative Research Group. Hyperglycemia and adverse pregnancy outcomes. N Engl J Med. 2008;358(19):1991-2002.

5. Catalano PM, McIntyre HD, Cruickshank JK, McCance DR, Dyer AR, Metzger BE, et al. The hyperglycemia and adverse pregnancy outcome study: associations of GDM and obesity with pregnancy outcomes. Diabetes Care. 2012:DC111790.

6. Crowther CA, Hiller JE, Moss JR, McPhee AJ, Jeffries WS, Robinson JS. Australian carbohydrate intolerance study in pregnant women trial G. Effect of treatment of gestational diabetes mellitus on pregnancy outcomes. N Engl J Med. 2005;352(24):2477-86.

7. Landon MB, Spong CY, Thom E, Carpenter MW, Ramin SM, Casey B, et al. A multicenter, randomized 
trial of treatment for mild gestational diabetes. $\mathrm{N}$ Engl $\mathbf{J}$ Med. 2009;361(14):1339-48.

8. Nanda S, Savvidou M, Syngelaki A, Akolekar R, Nicolaides KH. Prediction of gestational diabetes mellitus by maternal factors and biomarkers at 11 to 13 weeks. Prenat Diagn. 2011;31(2):135-41.

9. Maged AM, Moety GA, Mostafa WA, Hamed DA. Comparative study between different biomarkers for early prediction of gestational diabetes mellitus. J Matern Fetal Neonatal Med. 2014;27(11):1108-12.

10. Yeral MI, Ozgu-Erdinc AS, Uygur D, Seckin KD, Karsli MF, Danisman AN. Prediction of gestational diabetes mellitus in the first trimester, comparison of fasting plasma glucose, two-step and one-step methods: a prospective randomized controlled trial. Endocrine. 2014;46(3):512-8.

11. Nahum GG, Wilson SB, Stanislaw H. Early-pregnancy glucose screening for gestational diabetes mellitus. J Reprod Med. 2002;47(8):656-62.

12. National Institute for Health and Care Excellence. Diabetes in pregnancy: management of diabetes and its complications from preconception to the postnatal period. London: Clinical Guidelines, 2015.

13. American Diabetes Association. Standards of medical care in diabetes-2015 abridged for primary care providers. Clin Diabetes. 2015;33:97-111.

14. Gupta Y, Kalra B, Baruah MP, Singla R, Kalra S. Updated guidelines on screening for gestational diabetes. Int J Womens Health. 2015;7:539-50.

15. International association of diabetes and pregnancy study groups consensus panel. International association of diabetes and pregnancy study groups recommendations on the diagnosis and classification of hyperglycemia in pregnancy. Diabetes Care. 2010;33:676-82.

16. Committee on Practice Bulletins-Obstetrics. Practice Bulletin No. 137: Gestational diabetes mellitus. Obstet Gynecol. 2013;122(Pt 1):406-16.

17. Clausen TD, Mathiesen ER, Hansen T, Pedersen O, Jensen DM, Lauenborg $\mathrm{J}$, et al. Overweight and the metabolic syndrome in adult offspring of women with diet-treated gestational diabetes mellitus or type 1 diabetes. J Clin Endocrinol Metab. 2009;94:2464-70.

18. Kim SY, England JL, Sharma JA, Njoroge T. Gestational diabetes mellitus and risk of childhood overweight and obesity in offspring: a systematic review. Exp Diabetes Res. 2011;54:130-8.

19. McMillen IC, Robinson JS. Developmental origins of the metabolic syndrome: prediction, plasticity, and programming. Physiol Rev. 2005;85:571-633.

20. Clausen TD, Mathiesen ER, Hansen T, Pedersen O, Jensen DM, Lauenborg J, et al. High prevalence of type 2 diabetes and pre-diabetes in adult offspring of women with gestational diabetes mellitus or type 1 diabetes: the role of intrauterine hyperglycemia. Diabetes Care. 2008;31:340-6.

21. Li X, X Lu. Study on correlation between C-reactive protein and gestational diabetes mellitus. J Nanjing Med Univ. 2007;21(6):382-5.

22. Liu T, Fang Z, Yang D, Liu Q. Correlation between the inflammatory factors and adipocytokines with gestational diabetes mellitus and their change in puerperium. Zhonghua $\mathrm{Fu}$ Chan $\mathrm{Ke} \mathrm{Za}$ Zhi. 2012;47(6):436-9.

23. Rota S, Yildirim B, Kaleli B, Aybek H, Duma K, Kaptanoglu B. C-reactive protein levels in non-obese pregnant women with gestational diabetes. Tohoku J Exp Med. 2005;206:341-5.

24. Qiu C, Sorensen TK, Luthy DA, Williams MA. Prospective study of maternal serum C-reactive protein (CRP) concentrations and risk of gestational diabetes mellitus. Paediatr Perinat Epidemiol. 2004;18(5):37784.

25. Wolf M, Sauk J, Shah A, Smirnakis KV, JimenezKimble R, Ecker JL, et al. Inflammation and glucose intolerance: a prospective study of gestational diabetes mellitus. Diabetes Care. 2004;27:21-7.

26. Radaelli T, Varastehpour A, Catalano P, Hauguel-de Mouzon S. Gestational diabetes induces placental genes for chronic stress and inflammatory pathways. Diabetes. 2003;52(12):2951-8.

27. Ahi Z, Kariman N, Asl SZ, Shakeri N. Diagnostic value of $\mathrm{C}$-reactive protein in determining of gestational diabetes mellitus (GDM). Biosci Biotechnol Res Asia. 2016;13(2):743-50.

28. Mohan BS. C-reactive protein in early months of pregnancy as a screening test for gestational diabetes mellitus developing in later months of pregnancy. J Obstet Gynecol India. 2006;56:131-3.

29. Kirwan JP, Hauguel-De Mouzon S, Lepercq J, Challier JC, Huston-Presley L, Friedman JE, et al. TNF- $\alpha$ is a predictor of insulin resistance in human pregnancy. Diabetes. 2002;51(7):2207-13.

30. Retnakaran R, Hanley AJ, Raif N, Connelly PW, Sermer M, Zinman B. C reactive protein and gestational diabetes: the central role of maternal obesity. J Clin Endocrinol Metab. 2003;88(8):3507- 12

31. Frohlich M, Emhof A, Berg G, Hutchinson W, Pepys $\mathrm{M}$, Boeing $\mathrm{H}$, et al. Association between C-reactive protein and features of the metabolic syndrome. Diabetes Care. 2000;23:1835-9.

32. Cunningham FG, et al. Williams Obstetrics. $24^{\text {th }}$ ed. New York: Wendel T; 2014.

33. Syngelaki A, Visser GH, Krithinakis K, Wright A, Nicolaides KH. First trimester screening for gestational diabetes mellitus by maternal factors and markers of inflammation. Metabol. 2016;65(3):131-7.

34. Corcoran S, Achamallah N, John OL, Grainne K, Stafford P, Meaney S, et al. Can first trimester highsensitivity C-reactive protein and sex hormone binding globulin predict the onset of gestational diabetes? a prospective study of a high risk cohort. Am J Obstet Gynecol. 2016;214(1):S388.

Cite this article as: Farghaly TA, Helmy NA, Abbas AM, Ahmed AGM. C-reactive protein as a screening test for gestational diabetes mellitus in first-trimester of pregnancy: a prospective cohort study. Int J Reprod Contracept Obstet Gynecol 2018;7:4798-803. 